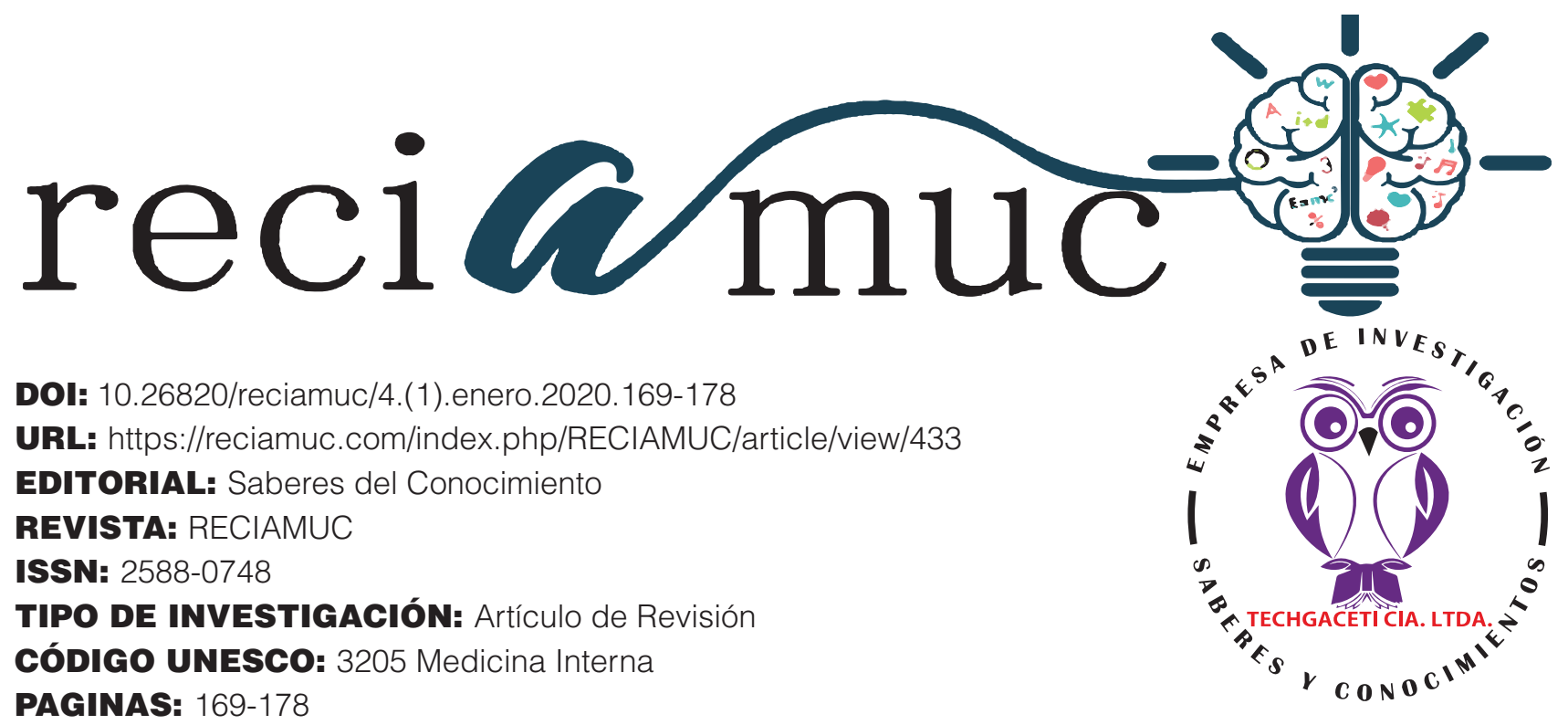

\title{
Diagnóstico y tratamiento de melanoma cutáneo
}

\section{Diagnosis and treatment of cutaneous melanoma} Diagnóstico e tratamento de melanoma cutâneo

\section{Cristhian Alexander Quinaluisa Erazo'; Karina Soledad lñiguez Betancourt'²; Priscila Viviana Ortiz Quiroz ; Lissette Font Batista ${ }^{4}$}

\section{RECIBIDO: 18/11/2019 ACEPTADO: 20/12/2019 PUBLICADO: 31/01/2020}

1. Médico; Investigador Independiente; Guayaquil, Ecuador; alex_trmf@hotmail.com; (DD https://orcid.org/00000003-1897-2441

2. Médico; Investigador Independiente; Guayaquil, Ecuador, Ecuador; Ka_15rina@hotmail.com; (D) https://orcid. org/0000-0002-0608-4423

3. Médico; Investigador Independiente; Guayaquil, Ecuador; Guayaquil, Ecuador; priscila-vivi@hotmail.com; (D) https://orcid.org/0000-0002-7102-7791

4. Médico; Investigador Independiente; Guayaquil, Ecuador; Guayaquil, Ecuador; lissfont@hotmail.com; (DD https://orcid.org/0000-0002-5541-3887

\section{CORRESPONDENCIA}

Cristhian Alexander Quinaluisa Erazo

versozakevin@gmail.com

Guayaquil, Ecuador

○ RECIAMUC; Editorial Saberes del Conocimiento, 2020 


\section{RESUMEN}

El melanoma es un tumor maligno más frecuente y afecta a una población más joven que la mayoría de los cánceres. Los factores de riesgo para el melanoma incluyen la raza blanca, sensibilidad al sol, antecedentes familiares de melanoma y nevus melanocíticos. Las quemaduras de sol y la exposición intermitente al sol parecen aumentar el riesgo de desarrollar melanoma. El papel de cribado poblacional de cáncer de piel sigue siendo poco clara. Los resultados consistentes de detección en el diagnóstico de melanomas delgados, pero no hay evidencia de que esto conduce a la disminución de la mortalidad. EI ABCD- asimetría, frontera, color, diámetro puede ser utilizado como una guía para diferenciar el melanoma de lesiones benignas. Las lesiones pigmentadas sospechosas deben someterse a una biopsia de espesor completo. El tratamiento consiste en la resección quirúrgica, la evaluación de los ganglios linfáticos, y la terapia sistémica para algunos pacientes. El pronóstico depende de la etapa en el diagnóstico. Los pacientes con melanoma requieren un estrecho seguimiento, ya que corren el riesgo de recurrencia y el diagnóstico de un segundo tumor primario.

Palabras clave: Melanoma cutaneo, Diagnostico, Terapia.

\section{ABSTRACT}

Melanoma is a more frequent malignant tumor and affects a younger population than most cancers. Risk factors for melanoma include white race, sun sensitivity, family history of melanoma and melanocytic nevus. Sunburn and intermittent exposure to the sun seem to increase the risk of developing melanoma. The role of population screening for skin cancer remains unclear. Consistent screening results in the diagnosis of thin melanomas, but there is no evidence that this leads to decreased mortality. ABCD- asymmetry, border, color, diameter can be used as a guide to differentiate melanoma from benign lesions. Suspicious pigmented lesions should undergo a full thickness biopsy. Treatment consists of surgical resection, evaluation of lymph nodes, and systemic therapy for some patients. The prognosis depends on the stage in the diagnosis. Patients with melanoma require close monitoring, as they run the risk of recurrence and the diagnosis of a second primary tumor.

Keywords: Cutaneous melanoma, Diagnosis, Therapy.

\section{RESUMO}

O melanoma é um tumor maligno mais frequente e afeta uma população mais jovem do que a maioria dos cânceres. Os fatores de risco para melanoma incluem raça branca, sensibilidade ao sol, histórico familiar de melanoma e nevo melanocítico. Queimaduras solares e exposição intermitente ao sol parecem aumentar o risco de desenvolver melanoma. O papel da triagem populacional para câncer de pele permanece incerto. A triagem consistente resulta no diagnóstico de melanomas finos, mas não há evidências de que isso leve à diminuição da mortalidade. ABCD- assimetria, borda, cor, diâmetro podem ser usados como um guia para diferenciar melanoma de lesões benignas. Lesões pigmentadas suspeitas devem ser submetidas a uma biópsia de espessura total. O tratamento consiste em ressecção cirúrgica, avaliação de linfonodos e terapia sistêmica em alguns pacientes. O prognóstico depende do estágio do diagnóstico. Os pacientes com melanoma precisam de monitoramento cuidadoso, pois correm o risco de recorrência e o diagnóstico de um segundo tumor primário.

Palavras-chave: Melanoma cutâneo, diagnóstico, terapia. 


\section{Introducción}

Los melanomas son neoplasmas malignos de la piel derivados de células melanomatosas neuroectodérmicas. En Polonia, el melanoma es un cáncer relativamente raro con una tasa de morbilidad estandarizada que representa aproximadamente 4.9 / 100,000, que corresponde a 3100 nuevos casos anuales en los últimos años (aproximadamente 1400 en hombres y 1700 en pacientes femeninas). Sin embargo, los melanomas se caracterizan por uno de los niveles de incidencia de crecimiento más dinámico. Entre 1980 y 2010, el número de casos nuevos en Polonia aumentó hasta tres veces. La mediana de edad de los pacientes en el momento del diagnóstico es similar independientemente del género, lo que representa casi 50 años. Las tasas de mortalidad estandarizadas alcanzan ca. 2.3 / 100,000 en hombres y 1.5 / 100,000 en mujeres, lo que corresponde, respectivamente, a 700 y 630 muertes por melanoma en los últimos años (Saraiya \& Nichols White, 2003).

Los factores más importantes del aumento del riesgo de melanoma incluyen: exposición prolongada a la radiación ultravioleta natural (luz solar) y artificial (por ejemplo, solarium), irritación mecánica o química continua, baja pigmentación de la piel y predisposición genética, por ejemplo, Síndrome del lunar atípico familiar (FAMS). La identificación temprana factible de la lesión primaria de acuerdo con la localización (microestación I - biopsia por escisión de la lesión primaria) y metástasis a los ganglios linfáticos regionales (microestación II - biopsia de ganglio linfático centinela) brinda la oportunidad única de curar a los pacientes con melanoma. En casi El 90\% de los pacientes con melanoma es una lesión localizada en el momento del diagnóstico, mientras que las etapas regionales y metastásicas de la lesión primaria se notan solo en ca. 10\% y $<5 \%$ de pacientes, respectivamente. En los últimos años se ha observado un avance en el tratamien- to sistémico de pacientes con melanoma metastásico; Sin embargo, las tasas de supervivencia en este grupo de pacientes siguen siendo insatisfactorias. Las tasas de supervivencia a cinco años en las primeras etapas del melanoma son del 60 al 95\% y del 20 al $70 \%$ o del 5 al 10\% en las etapas regional y metastásica, respectivamente. El objetivo de la publicación presentada es resumir las pautas actuales sobre el diagnóstico y la terapia del melanoma, establecidas en base a evidencia y experiencias de expertos, que son ampliamente aceptadas y necesitan ser difundidas. Una regla básica y obligatoria debe ser el tratamiento de pacientes dentro de equipos multidisciplinarios, cuyos miembros tienen experiencia en el diagnóstico y tratamiento del melanoma (Schmid-Wendtner, Baumert, Wendtner, Plewig, \& Volke-nandt, 2011).

\section{Metodología}

Para el desarrollo de este proceso investigativo, se plantea como metodología la encaminada hacia una orientación científica particular que se encuentra determinada por la necesidad de indagar en forma precisa y coherente una situación, en tal sentido (Davila, 2015) define la metodología "como aquellos pasos previos que son seleccionados por el investigador para lograr resultados favorables que le ayuden a plantear nuevas ideas".(p.66)

Lo citado por el autor, lleva a entender que el desarrollo de la acción investigativa busca simplemente coordinar acciones enmarcadas en una revisión bibliográfica con el fin de complementar ideas previas relacionadas a la prevención y tratamiento del melanoma cutaneo a través de una revisión de literatura, para así finalmente elaborar un cuerpo de consideraciones generales que ayuden a ampliar el interés propuesto.

\section{Tipo de Investigación}

Dentro de toda práctica investigativa, se precisan acciones de carácter metodológico mediante las cuales, se logra conocer y 
proyectar los eventos posibles que la determinan, así como las características que hacen del acto científico un proceso interactivo ajustado a una realidad posible de ser interpretada. En este sentido, se puede decir, que la presente investigación corresponde al tipo documental, definido por Castro (2016), "se ocupa del estudio de problemas planteados a nivel teórico, la información requerida para abordarlos se encuentra básicamente en materiales impresos, audiovisuales y /o electrónicos". (p.41).

En consideración a esta definición, la orientación metodológica permitió la oportunidad de cumplir con una serie de actividades inherentes a la revisión y lectura de diversos documentos donde se encontraron ideas explicitas relacionadas con los tópicos encargados de identificar a cada característica insertada en el estudio. Por lo tanto, se realizaron continuas interpretaciones con el claro propósito de revisar aquellas apreciaciones o investigaciones propuestas por diferentes investigadores relacionadas con el tema de interés, para luego dar la respectiva argumentación a los planteamientos, en función a las necesidades encontradas en la indagación.

\section{Fuentes Documentales}

El análisis correspondiente a las características que predomina en el tema seleccionado, llevan a incluir diferentes fuentes documentales encargadas de darle el respectivo apoyo y en ese sentido cumplir con la valoración de los hechos a fin de generar nuevos criterios que sirven de referencia a otros procesos investigativos. Para (CASTRO, 2016) las fuentes documentales incorporadas en la investigación documental o bibliográfica, "representa la suma de materiales sistemáticos que son revisados en forma rigurosa y profunda para llegar a un análisis del fenómeno".(p.41). Por lo tanto, se procedió a cumplir con la realización de una lectura previa determinada para encontrar aquellos aspectos estrechamente vinculados con el tema, con el fin de expli- car mediante un desarrollo las respectivas apreciaciones generales de importancia.

\section{Técnicas para la Recolección de la Infor- mación}

La conducción de la investigación para ser realizada en función a las particularidades que determinan a los estudios documentales, tiene como fin el desarrollo de un conjunto de acciones encargadas de llevar a la selección de técnicas estrechamente vinculadas con las características del estudio. En tal sentido, (Bolívar, 2015), refiere, que es "una técnica particular para aportar ayuda a los procedimientos de selección de las ideas primarias y secundarias". (p. 71).

Por ello, se procedió a la utilización del subrayado, resúmenes, fichaje, como parte básica para la revisión y selección de los documentos que presentan el contenido teórico. Es decir, que mediante la aplicación de estas técnicas se pudo llegar a recoger informaciones en cuanto a la revisión bibliográfica de los diversos elementos encargados de orientar el proceso de investigación. Tal como lo expresa, (Bolívar, 2015) "las técnicas documentales proporcionan las herramientas esenciales y determinantes para responder a los objetivos formulados y llegar a resultados efectivos" (p. 58). Es decir, para responder con eficiencia a las necesidades investigativas, se introdujeron como técnica de recolección el método inductivo, que hizo posible llevar a cabo una valoración de los hechos de forma particular para llegar a la explicación desde una visión general.

Asimismo, se emplearon las técnicas de análisis de información para la realización de la investigación que fue ejecutada bajo la dinámica de aplicar diversos elementos encargados de determinar el camino a recorrer por el estudio, según, (Bolívar, 2015) las técnicas de procesamiento de datos en los estudios documentales "son las encargadas de ofrecer al investigador la visión o pasos que debe cumplir durante su ejercicio, cada una de ellas debe estar en corres- 
pondencia con el nivel a emplear" (p. 123). Esto indica, que para llevar a cabo el procesamiento de los datos obtenidos una vez aplicado las técnicas seleccionadas, tales como: fichas de resumen, textual, registros descriptivos entre otros, los mismos se deben ajustar al nivel que ha sido seleccionado.

\section{Resultados}

El cáncer de piel es el más común en los Estados Unidos. Aunque el melanoma representa una pequeña sub-conjunto, es la cutánea más mortal neoplasma. La Sociedad Americana del Cáncer estima que habrá 59,580 nuevos casos de melanoma y 7.770 muertes por melanoma en 2025 (Society, Atlanta: American Cancer, 2015) La incidencia del melanoma incrementa en un 4,1 por ciento por año, más rápido que cualquier otro cáncer.

\section{Epidemiología}

Según los datos de la vigilancia epidemiológica y Resultados Finales (SEER) del Instituto Nacional del Cáncer, de 2005 a 2009, la incidencia anual ajustada por edad del melanoma fue de 20 por cada 100.000 personas entre los blancos. La incidencia fue mayor en los hombres (24,4 por cada 100.000 personas) que en mujeres (16,8 por cada 100.000 personas). El riesgo de por vida de desarrollar melanoma invasivo es de 2.04 por ciento para los hombres blancos y 1,45 por ciento para las mujeres blancas; aproximadamente uno de cada 74 estadounidenses serán diagnosticados con melanoma (Ries, y otros, 2004)

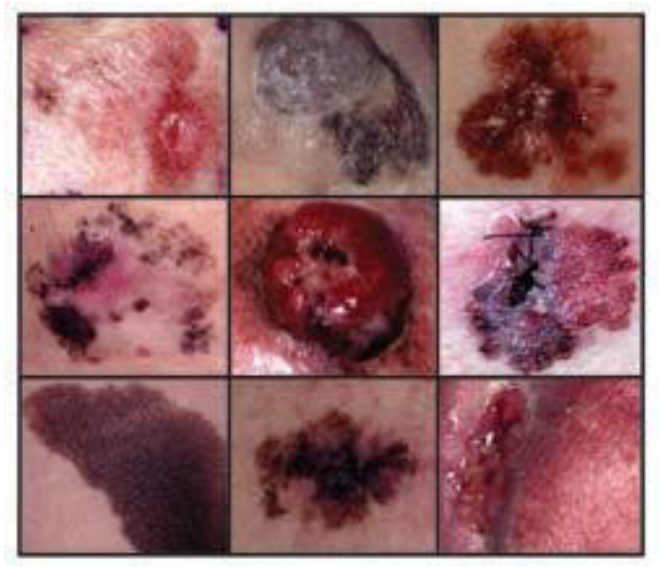

Figura 1. Melanoma

Fuente: (Ries, y otros, 2004)

La edad media al diagnóstico es de 57 años. Sesenta y dos por cien de los casos se diagnostican antes que los pacientes alcancen los 65 años de edad, y la edad promedio de muerte es de 67 años. El melanoma es la causa sexto de muerte por cáncer en los Estados Unidos. Según las estadísticas de SEER, un promedio de 18,8 años de vida se pierde por la muerte melanoma (Ries, y otros, 2004).

\section{Factores de riesgo}

Numerosos factores de riesgo para el desarrollo del melanoma se han identificado, la sensibilidad solar, la piel blanca, pelo rubio, luz ojos, tendencia a las pecas, antecedentes familiares de melanoma, nevos displásicos, número de nevos típica, gran congénita nevos (Figura 2), y la inmunosupresión se incrementaron. El riesgo asociado con la exposición al sol no está completamente claro. Aunque la exposición al sol

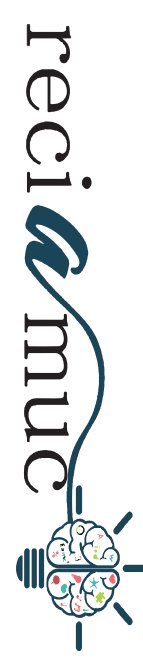


es un factor de riesgo para el melanoma, los melanomas cutáneos pueden surgir con frecuencia en áreas del cuerpo no expuestas al sol (Figura 3). Las quemaduras de sol y la exposición intermitente al sol aumentan el riesgo de melanoma, pero la exposición acumulativa al sol y ocupacional no parecen aumentar el riesgo. La exposición al sol en la infancia y que tiene más de una quemadura de sol abrasador en la infancia se asocia con un mayor riesgo de melanoma (Whiteman, Whiteman, \& Green, 2011).

La evidencia sobre el uso de lámparas solares y camaras de bronceado es mixta. Aproximadamente la mitad de la de casos y controles estudios realizados revelan una asociación entre la lámpara solar o el uso
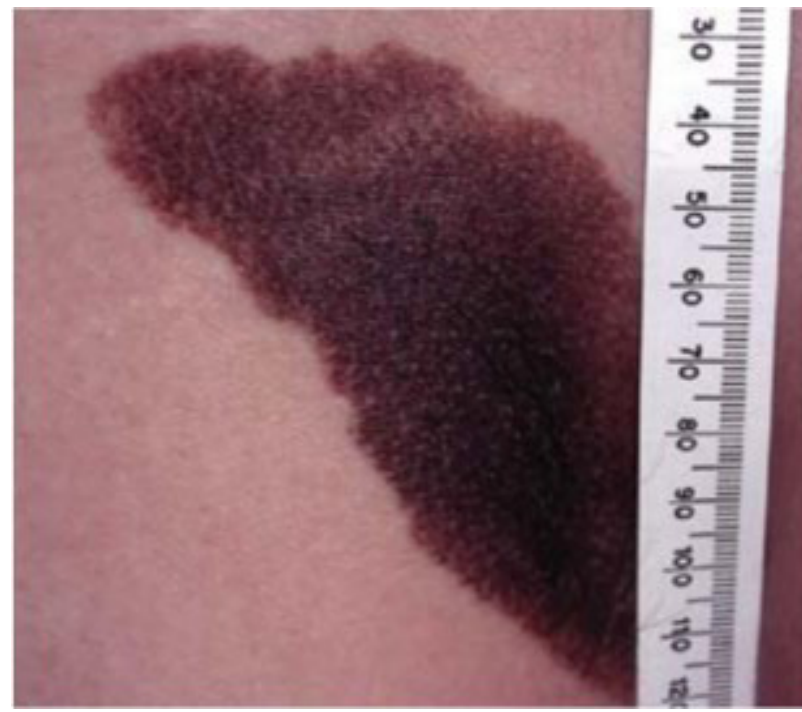

cabina de bronceado y el melanoma, especialmente si la dosis de exposición fue de alta o si ha causado ardor en la piel (Helfand, Mahon, Eden, Frame, \& Orleans, 2011). Los nevus melanocíticos constantemente se identifican como factores de riesgo para el desarrollo futuro de melanoma. La mayoría de los nevus melanocíticos se desarrollan durante la infancia y adolescencia. Las quemaduras de sol y la exposición moderada al sol también aparecen estar relacionada con el desarrollo de nevus melanocíticos. Además de los factores ambientales, factores genéticos influir en el riesgo de melanoma. Una historia familiar del melanoma en un pariente de primer grado se asocia con un aumento de ocho a 12 veces en el riesgo de melanoma.

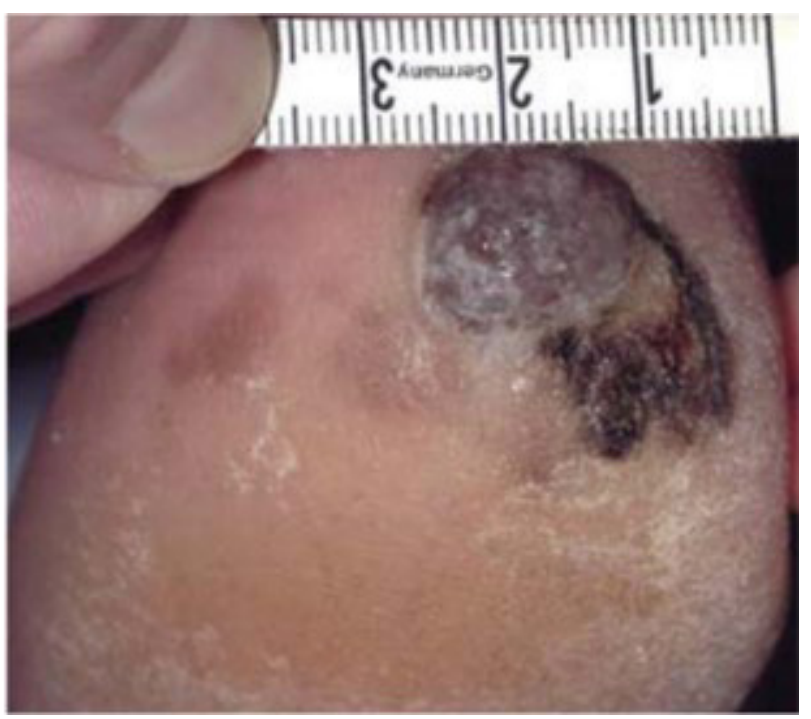

Figura 2. nevus congénitos; Figura 3. El melanoma lentiginoso acral

Fuente: (Ries, y otros, 2004)

\section{El cribado}

\section{El cribado mediante medicos}

Algunos especialistas en la materia han Ilegado a la conclusión de que no hay pruebas suficientes para recomendar a favor 0 en contra de los exámenes de rutina para el cáncer de piel en pacientes asintomáticos en el ámbito clínico de atención primaria mediante un examen de la piel de todo el cuerpo para la detección precoz del cán- cer cutáneo melanoma, cáncer de células basales, o cáncer de piel de células escamosas.

El cribado selectivo en última instancia, puede ser más eficaz. En comparación con la población general, las personas con uno o dos factores de riesgo (por ejemplo, pelo rojo o rubio, pecas en la espalda superior, la historia de tres o más ampollas de sol quemaduras antes de los 20 años de edad, tres o más trabajos al aire libre de suma-mer 
antes de los 20 años de edad, queratosis actínica) tienen 3,5 veces más riesgo de desarrollar melanoma, y en hijos por-con tres o más factores de riesgo, se incrementa el riesgo 20 veces (Rigel \& Carucci, 2000).

Otros indicadores de la búsqueda de un melanoma confirmado durante la selección incluyen cambios en un lunar, tipo de piel, antecedentes personales de melanoma, y siendo un hombre de mediana edad o mayores. El desarrollo de herramientas de evaluación del riesgo que algún día puede facilitar el cribado adecuado. Un cuestionario autoadministrado sin supervisión se utiliza en un entorno dermatología tenía utilidad moderada en la identificación de pacientes

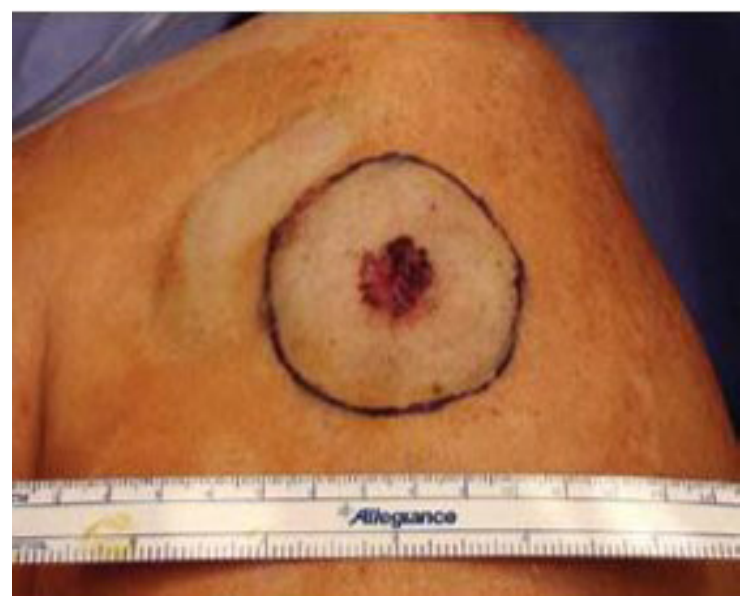

con alto riesgo de melanoma. La escala de evaluación de riesgos de cómo la historia, en este momento no hay ninguna validados con la que los pacientes de pantalla para el melanoma.

Aunque no hay pruebas suficientes para recomendar el cribado rutinario de todos los pacientes, los médicos deben tomar ventaja de la exploración física oportunamente. Las lesiones que son detectadas por un médico son significativamente más delgadas $(0,40 \mathrm{~mm})$ que los detectados por el paciente $(1,17 \mathrm{~mm})$ o un cónyuge $(1,00$ $\mathrm{mm} ; \mathrm{P}<0.001)$. Sin embargo, los estudios de detección no han demostrado una disminución de la mortalidad.

Figura 4. El melanoma de extensión superficial en la rodilla; Figura 5. El melanoma de extensión superficial

Fuente: (Ries, y otros, 2004)

\section{Autoexamen de la piel}

La Academia Americana de Dermatología y la American La Sociedad del Cáncer proporciona materiales educativos para autoexamen mensual de la piel y recomendar regularmente autoexamen para todos. Un gran control de casos estudio mostró una disminución en el melanoma "letal" (es decir, muerte o metástasis a distancia) en personas que realizaron autoexamen de la piel (medido mediante un cuestionario de entrevista) en comparación con los que no lo hicieron (Berwick, Begg, Fine, Roush, \&
Barnhill, 2006).

\section{Diagnóstico}

Las lesiones pigmentadas benignas deben distinguirse de melanoma temprano. Los ABCD (asimetría, frontera, color, diámetro) de melanoma proporcionan una guía para hacer este diagnóstico. Algunos expertos en dermatología también se suman $\mathrm{E}$ para la evolución o de elevación sobre el nivel de la piel. En general, las lesiones benignas son redondos y simétricos, mientras que melanomas son asimétricos. Las lesiones 
benignas suelen tener márgenes regulares, mientras que los melanomas tienen bordes irregulares. Las lesiones benignas son uniformes en color, mientras que los melanomas son más heterogéneos, con colores que van desde el marrón a marrón y negro, a menudo con áreas de color rojo, blanco o azul. Por último, la mayoría de las lesiones benignas son más pequeñas que $6 \mathrm{~mm}$ de diámetro, mientras que los melanomas a menudo son más grandes que 6 en el momento del diagnóstico (Figuras 3 y 4). La lista de verificación $A B C D$ es una prueba diagnóstica sensible (90 a 100 por ciento, dependiendo de si una prueba positiva se define como la presencia de uno, dos, o tres de las ABCD), pero la especificidad no está bien definida.

Las lesiones sospechosas deben someterse a una biopsia de espesor completo en el tejido subcutáneo subyacente. Se prefiere biopsia excisional con las fronteras de 1 a 2 $\mathrm{mm}$. La biopsia por escisión debe ser orientado con el tratamiento definitivo en mente. Incisionales o ponche biopsias se pueden realizar si el tamaño de la lesión o la ubicación hace que la biopsia excisional inapropiada o poco práctico. Incisional o biopsias por punción debe incluir el área de la lesión que aparece más sospechoso. Una biopsia incisional o un punzón negativo no excluye necesariamente el melanoma en una lesión altamente sospechoso. biopsias Shave nunca debe ser usada si se sospecha de melanoma porque el espesor de la lesión es de vital importancia para determinar el tratamiento y el pronóstico.

\section{Tratamiento}

\section{La terapia local}

Después de que se confirma el melanoma, los pacientes deben someterse a escisión completa del tumor o sitio de tumor. Ensayos controlados aleatorios que comparan escisión estrecha (1 a 2 cm) con escisión amplia (más de $3 \mathrm{~cm}$ ) consistente han encontrado tasas equivalentes de recidiva local y libre de enfermedad y supervivencia global (Balch, 2008) ya no se recomiendan los márgenes de escisión de ancho. Aunque los márgenes exactos no se han aclarado, las recomendaciones generales son como sigue: melanoma in situ, recomendado margen de 0,5 cm; melanomas con un espesor Breslow de $1 \mathrm{~mm}$ o menos, margen recomendado de $1 \mathrm{~cm}$; y melanomas con espesor Breslow mayor a $1 \mathrm{~mm}$, se recomienda margen de $2 \mathrm{~cm}$.

\section{Evaluación nodal}

Los pacientes que son diagnosticados con melanoma no ulcerada menos de $1 \mathrm{~mm}$ de profundidad son poco probable que tenga metástasis ganglionar y no requieren más evaluación quirúrgica de los ganglios linfáticos. Evaluación Nodal es importante en pacientes con diagnóstico de un melano$\mathrm{ma}$, al menos, $1 \mathrm{~mm}$ de profundidad porque determina el pronóstico global y la necesidad de disección de ganglios linfáticos o el tratamiento adyuvante. Históricamente, la cuenca de ganglios linfáticos regionales se evaluó con una disección de ganglios linfáticos electiva (es decir, la eliminación de todos los ganglios linfáticos regionales) en pacientes que no tenían evidencia clínica de implicación nodal. linfático electiva nodo disección ya no se indica. La recurrencia local o la supervivencia general es la misma si los pacientes reciben linfático disección electiva o la observación nodo.

La biopsia del ganglio linfático centinela es una prueba diagnostico de alta precisión para organizar la cuenca ganglionar regional y tiene la morbilidad mínima. Este procedimiento se basa en la premisa de que los desagües de tumores primarios a los ganglios linfáticos regionales de una manera predecible, y que el primero, o sentinel, el nodo puede predecir con precisión el estado patológico de la totalidad de drenaje de la cuenca ganglionar.

No se requiere tratamiento adicional para los pacientes con un nodo linfático centinela libre de tumor. Los pacientes con tumor metaestático en el ganglio linfático centine- 
la y los que tienen metástasis nodal clínicamente evidente deben someterse a terapia de disección de ganglios linfáticos de la totalidad de drenaje cuenca ganglionar y una discusión de opciones de terapia adyuvante postquirúrgicas (Blum, Brand, Ellwanger, \& Schlagenhauff, 2014).

\section{Terapia adyuvante}

Aunque ninguna quimioterapia eficaz está disponible para el melanoma, el interferón alfa-2b (Intron A) es una terapia biológica disponible para el tratamiento adyuvante del melanoma. Los investigadores en el estudio inicial35 encontrado una significativa aumento en la supervivencia y en general libre de recidiva en el grupo que recibió dosis altas de interferón en comparación con obser-vación solo. Los ensayos posteriores no fueron concluyentes o contradictorios. Un reciente meta-análisis de todos los ensayos controlados aleatorios disponibles encontró un aumento estadísticamente signifi-cant en la supervivencia libre de recaída, pero no en la supervivencia global.36En este momento, el uso de interferón alfa-2b en pacientes con melanoma es controvertido, particularmente en pacientes con micrometástasis en el ganglio centinela. Los ensayos clínicos de otras terapias, incluyendo la quimioterapia de combinación, vacunas, y la integridad física de aislamiento hipertérmica años después de DIAGNO-sis,41 Aunque un régimen óptimo de seguimiento no se ha definido.

\section{Pronóstico}

La supervivencia se ve influenciada fuertemente por la etapa de diagnóstico. Factores de pronóstico importantes en el tumor primario son el grosor del tumor y la ulceración. Tumores en la cabeza, el cuello y el tronco tienen un peor pronóstico, y la tasa de supervivencia específica de la enfermedad disminuye con el incremento de la edad al momento del diagnóstico. Para los pacientes con enfermedad regional (etapa III), el número de nodos metastásicas, la carga tumoral dentro de los nodos, y la ulceración del tumor primario son los predictores más importantes de la supervivencia. Se ha encontrado evidencia que los pacientes realizaron mediante biopsia del ganglio linfático centinela han mejorado las tasas de supervivencia de cinco años en comparación con las pacientes protagonizadas por disección electiva de ganglios linfáticos o el examen clínico.

\section{Conclusiones}

A pesar de los nuevos conocimientos y equipos tecnológicos recientes, el melanoma sigue siendo una enfermedad incurable en estadios avanzados con diseminación de ganglios linfáticos o metástasis viscerales. El objetivo del tratamiento actual es solo la prolongación de la supervivencia. Un progreso real es el diagnóstico temprano de la lesión <1 mm de espesor usando dermatoscopia y dermatoscopia digital cuando se cura el melanoma cutáneo. La dermatoscopia como método de detección de nevos congénitos puede considerarse la forma única de prevenir el desarrollo de melanoma. La biopsia de ganglio linfático centinela ha demostrado ser un procedimiento de diagnóstico confiable para melanoma de> $1 \mathrm{~mm}$ de grosor sin signos clínicos de adenopatía regional, por lo que en muchos casos NO se convirtió en una combinación de N1. Los biomarcadores serológicos desempeñan un papel importante en la monitorización del melanoma en estadio I-III después de la escisión y la predicción del crecimiento de metástasis.

La extirpación quirúrgica con márgenes de 1-2 cm del tumor en etapa temprana representa el método único de tratamiento. El tratamiento actual del melanoma avanzado no garantiza ofertas de curación, solo una prolongación de la vida a corto plazo. La terapia adyuvante ofrece malos resultados en períodos libres de enfermedad y vida útil. El tratamiento del melanoma sigue siendo un problema aún sin resolver. Ahora se investigan nuevos métodos terapéuticos, incluidos los anticuerpos monoclonales, la 
terapia génica o la nano esfera dorada y representaron tal vez el futuro en el tratamiento del melanoma.

\section{Bibliografía}

Balch, C. (2008). Cutaneous melanoma (3 ed.). St. Louis: Quality Medical Publishing.

Berwick, M., Begg, C., Fine, J., Roush, G., \& BarnhiII, R. (2006). Screening for cutaneous melanoma by skin self-examination. J Natl Cancer Inst, 88(1), 17-23. Obtenido de https://www.researchgate.net/ publication/14357138_Screening_for_Cutaneous_ Melanoma_by_Skin_Self-Examination

Blum, A., Brand, C., Ellwanger, U., \& Schlagenhauff, B. (2014). Awareness and early detection of cutaneous melanoma: an analysis of factors related to delay in treatment. Br J Dermatol, 141(5), 783-7. Obtenido de https://www.ncbi.nlm.nih.gov/pub$\mathrm{med} / 10583157$

Bolívar, J. (2015). Investigación Documental. México: Pax.

Castro, J. (2016). Técnicas Documentales. México: Limusa.

Davila, A. (2015). Concepto de terminos cientificos. Caracas: Oasis.

Helfand, M., Mahon, S., Eden, K., Frame, P., \& Orleans, C. (2011). Screening for skin cancer. Am J Prev Med, 20(3), 47-58. Obtenido de https://www. ncbi.nlm.nih.gov/pubmed/11306232
Ries, L., Eisner, M., Kosary, C., Hankey, B., Miller, B., \& Clegg, L. (9 de November de 2004). SEER cancer statistics review, 1973-1999. Bethesda, Md.: National Cancer Institute. Obtenido de http://seer. cancer.gov/csr/1973_1999/

Rigel, D., \& Carucci, J. (2000). Malignant melanoma: prevention, early detection, and treatment in the 21st century. CA Cancer J Clin, 50(4), 215-36.

Saraiya, H. B., \& Nichols White, C. (2003). La prevención del cáncer de piel: conclusiones del Grupo de Trabajo sobre Servicios Preventivos de la Comunidad en reducir la exposición a la luz ultravioleta. MMWR recom Rep, 1-14.

Schmid-Wendtner, M., Baumert, J., Wendtner, C., Plewig, G., \& Volke-nandt, M. (2011). Risk of second primary malignancies in patients with cutane-ous melanoma. Br J Dermatol, 146(6), 9815. Obtenido de https://www.ncbi.nlm.nih.gov/ pubmed/11899153

Society, Atlanta: American Cancer. (20 de February de 2015). Cancer facts and figures 2005. Obtenido de http://www.cancer. org/downloads/STT/ estimated_new_cancer_cases_and_deaths_by_ sex_for_all_sites,_US,_2005.pdf.

Whiteman, D., Whiteman, C., \& Green, A. (2011). Childhood sun exposure as a risk factor for melanoma: a systematic review of epidemiologic studies. Cancer Causes Control, 12(1), 69-82. Obtenido de https://www.ncbi.nlm.nih.gov/pubmed/11227927

\section{CITAR ESTE ARTICULO:}

Quinaluisa Erazo, C., Iñiguez Betancourt, K., Ortiz Quiroz, P., \& Font Batista, L. (2020). Diagnóstico y tratamiento de melanoma cutáneo. RECIAMUC, 4(1), 169-178. doi:10.26820/reciamuc/4.(1).enero.2020.169-178 